The Astrophysical, Journal, Vol. 154, October 1968

\title{
RAPID CHANGES IN THE OPTICAL INTENSITY AND RADIAL VELOCITIES OF THE X-RAY SOURCE SCO X-1
}

\author{
J. A. Westrhal, Allan Sandage, and Jerome Kristian \\ Mount Wilson and Palomar Observatories, Carnegie Institution of Washington, \\ California Institute of Technology \\ Received March 25, 1968
}

\begin{abstract}
The optical flux of Sco X-1 was continuously monitored on five separate nights between April and June 1967. Pulse counting was used with sampling intervals of $5 \mathrm{sec}$ on the 100- and 200-inch telescopes and $15 \mathrm{sec}$ on the 60 -inch reflector. The error due to photon statistics per sample interval was smaller than $0.007 \mathrm{mag}$ in all cases. The data were processed entirely by computer, using a variety of special digital techniques.

Rapid optical flickering with amplitudes of $\sim 0.02 \mathrm{mag}$ on a time scale of minutes was found on all five nights. These high-frequency fluctuations are superposed on slower continuous variations with amplitudes $\sim 0.15 \mathrm{mag}$, and on occasional bursts of $0.2 \mathrm{mag}$ which last about $10 \mathrm{~min}$ with very short rise and decay times.

Radial velocities have been measured by a digital method of cross-correlation of microphotometer records of the original photographic plates (Westphal 1966). Velocity changes of about $100 \mathrm{~km} \mathrm{sec}^{-1}$ were found on two different singly trailed plates, each of 3 hours' duration. The hydrogen lines and He II ( $\lambda 4686)$ change in opposite directions, similar to the extar Cyg X-2, suggesting, but not proving, binary motion. Systematic changes of velocity from night to night are set out in Table 2 .

The distance to Sco X-1 is estimated to be $D \simeq 500$ pc by three methods, based on the strength of interstellar Ca II K, and on the old nova hypothesis. A firm lower limit of $D \geq 300$ pc seems well established.

The total X-ray power is $\simeq 2 \times 10^{37} h^{2} \mathrm{ergs} \mathrm{sec}^{-1}$, where $h$ is the distance in units of $500 \mathrm{pc}$. If the $\mathrm{X}$ ray energy is due to bremsstrahlung, this level requires Sco X-1 to continuously pump energy into the hot plasma, replenishing the entire energy store of gas in the cooling time of $t_{c}=3 \times 10^{-15} h^{-1} R^{3 / 2} \mathrm{sec}$ where $R$ is the radius (in centimeters) of the plasma system. Reasonable values of $10^{11} \mathrm{~cm}<R<10^{12} \mathrm{~cm}$ give $100 h^{-1}<t_{c}<3000 h^{-1}$ sec, which, remarkably, is the characteristic time of the light flicker. Mechanical energy due to some type of low- $Q$ oscillation of the system, similar in many respects to that observed in the Sun, seems capable of providing $10^{37} \mathrm{ergs} \mathrm{sec}^{-1}$ to the plasma gas. The crucial experiment remains to simultaneously monitor the optical and the X-ray flux over periods of several hours. Detailed correlation of fluctuations is not expected, but the mean X-ray flux might plausibly be expected to vary in periods of fractions of an hour as the mean amplitude of the optical fluctuations changes.
\end{abstract}

\section{INTRODUCTION}

Knowledge of the optical properties of X-ray sources is of obvious importance in constructing plausible physical models of X-ray production. Among these properties is the pronounced flickering of the intensity of Sco X-1 on a time scale of minutes. The phenomenon, discovered during the optical identification program in October 1966 (Sandage et al. 1966), seemed of such relevance that we began a special series of observations in April 1967 to determine the nature of the short-term fluctuations.

We report here the results of five nights of continuous photoelectric monitoring of the optical flux, using special digital apparatus on the 60-, 100-, and 200-inch reflectors. The equipment was operated with sampling times of either 5 or $15 \mathrm{sec}$, and with sensitivities such that the photometric errors due to photon statistics in each integration interval are all considerably less than \pm 0.01 mag. Results of a similar study of Cyg X-2 on one night have already been published (Kristian, Sandage, and Westphal 1967).

Other photoelectric observations have been published by several workers, including Sandage et al. (1966), Mumford (1966b), Mook (1967), Stepien (1968), and Hiltner and 
Mook $(1967 a, b)$. The extensive compilation of Hiltner and Mook is of particular value for study of the variations on a time scale of hours and days. The primary relevance of our data is for shorter time intervals.

\section{PHOTOMETRY}

We have used a single-channel technique, in contrast to the more advanced doublechannel procedure which was later employed for Cyg X-2 (Kristian et al. 1967). Pulse counting was used throughout. Precise integration times were controlled by the internal crystal oscillators of the data-handling systems that were designed by E. W. Dennison's electronics group for the 100 - and 200 -inch reflectors. On the 60 -inch, a mechanical timer controlled by a synchronous motor began each integration period, whose duration was determined by the internal crystal timer of the commercial counter.

A variety of photoelectric equipment was used on the five nights of observation. Different photoelectric cells, used with or without a blue filter, were chosen such that adequate photon statistics were obtained in times as short as several seconds on each of the three telescopes. The telescopes and auxiliary equipment for each night are summarized in Table 1.

On the first night-April 3, 1967-the flux was sampled every 5 sec with a 1P21 plus $B$ filter using the 100 -inch reflector. Of the sampling time 2 sec were used for counting and the remaining $3 \mathrm{sec}$ for mechanically recording the pulse count with an on-line IBM card punch. The counter was started automatically by an electronic switch to begin the counting interval. This instrumental feature insured that the epoch of successive sampling intervals advanced at a uniform rate - an important requirement for the subsequent Fourier analysis of the data.

Sky readings were made periodically and were later subtracted by computer from each 5-sec data sample. Interpolated values were calculated from a polynomial fitted to the sky counts. The net count is plotted in Figure 1, which shows the computer-drawn digital record covering the 2.5 -hour monitoring interval. The sky conditions were nearly perfect as regards seeing and transparency. The sky-blocking diaphragm of 15".0 was at least ten times larger than the seeing image for that night. The digital record in Figure 1 , composed of 1800 individual 5-sec data points, has been numerically smoothed by a Gaussian filter of 15 -sec equivalent width, following a modified procedure described elsewhere (Westphal 1966).

As in Cyg X-2, small-amplitude fluctuations are present with a time scale of the order of minutes. These are superposed on a longer-term fluctuation whose time scale is about $30 \mathrm{~min}$. To investigate the high-frequency fluctuations more completely, we have separated the high- and low-frequency components of the light curve as follows. The original data, stored on punched cards, were heavily filtered to remove the high-frequency component. The equivalent width of this filter was $162 \mathrm{sec}$. The resulting low-frequency record, which gives the base level about which the short-period fluctuations occur, was then subtracted by computer from the original data, and the difference, which now contains only the high-frequency component, is displayed at the bottom of Figure 1 .

Most of the fluctuations are real: the error due to photon statistics for this lightly filtered record is about fifteen times smaller than the peak-to-peak amplitude of the fluctuations. Furthermore, continuous monitoring of a control star on another night of comparable transparency, described later and shown in Figure 3, shows that variations in transparency are negligible at the level we are discussing.

The high-frequency fluctuations have been found on all nights. The average peak-topeak amplitudes are about $0.015 \mathrm{mag}$, but at times they reach amplitudes of $0.05 \mathrm{mag}$ or more. To the eye, the phenomenon appears to be almost periodic, but Fourier analysis, as well as the equivalent autocorrelation, accomplished in this case by lagging duplicate tracings on transparent paper and inspecting the fit, show that the oscillations are not 
TABLE 1

\begin{tabular}{|c|c|c|c|c|c|c|c|c|c|c|c|}
\hline 1967 (U.T.) & $\begin{array}{l}\text { Telescope } \\
\text { (inch) }\end{array}$ & $\begin{array}{l}\text { Cell and } \\
\text { Filter }\end{array}$ & $\lambda(\mu)$ & $\begin{array}{l}\text { Integration } \\
\text { Time } \\
\text { (sec) }\end{array}$ & $\begin{array}{c}\text { Sample } \\
\text { Interval } \\
(\mathrm{sec})\end{array}$ & $\begin{array}{l}\text { Total-Run } \\
\text { Time }\end{array}$ & $\begin{array}{c}\text { Statistical } \\
\text { Error per } \\
\text { Point (mag) }\end{array}$ & $V^{*}$ (mag) & $B-V$ & $U-B$ & $\begin{array}{c}\text { Starting } \\
\text { Time } \\
\text { (U.T.) }\end{array}$ \\
\hline $\begin{array}{l}\text { April } 3 \\
\text { April } 13 \\
\text { May } 12 . \\
\text { June } 3 \\
\text { June } 5 .\end{array}$ & $\begin{array}{r}100 \\
200 \\
60 \\
100 \\
100\end{array}$ & $\begin{array}{l}\text { 1P21+B } \\
\text { 1P21+B } \\
\text { S20+none } \\
\text { S20+none } \\
\text { S20+none }\end{array}$ & $\begin{array}{r}0.40-0.55 \\
40-.55 \\
.32-.85 \\
.32-.85 \\
0.32-0.85\end{array}$ & $\begin{array}{r}2 \\
4 \\
10 \\
2 \\
2\end{array}$ & $\begin{array}{r}5 \\
5 \\
15 \\
5 \\
5\end{array}$ & $\begin{array}{lll}2^{\mathrm{h}} & 28^{\mathrm{m}} & 20^{\mathrm{s}} \\
3 & 30 & 25 \\
4 & 27 & 30 \\
2 & 44 & 35 \\
2 & 37 & 55\end{array}$ & $\begin{array}{r}0.0065 \\
.0036 \\
0058 \\
.0060 \\
0.0056\end{array}$ & $\begin{array}{l}12.25 \\
12.95 \\
12.38 \\
12.41 \\
12.36 \\
12.29 \dagger\end{array}$ & $\begin{array}{r}0.42 \\
.37 \\
.21 \\
.10 \\
07 \\
0.19\end{array}$ & $\begin{array}{l}-0.65 \\
-\quad 76 \\
-\quad .81 \\
-.83 \\
-0.83\end{array}$ & $\begin{array}{l}08.22 \\
06: 27 \\
07.14 \\
07: 09\end{array}$ \\
\hline
\end{tabular}

* An additional non-monitored night with the $200-1 n c h$ gave $V=12.97 \mathrm{mag}, B-V=0.21, U-B=-0.73$ for March $8,1967$.

$\dagger$ All $V$ magnitudes and colors are for beginning of run except this value, which was at end of run. 
phasable. However, neither are they completely white, because all frequencies are not present with equal amplitudes. Visual inspection of the records suggests that short wave trains of a dominant frequency may be involved.

Of particular interest in Figure 1 is the burst of about 10-min duration with an amplitude of $0.20 \mathrm{mag}$ centered at the $2800-\mathrm{sec}$ time marker. Note the longer rise time of the burst compared with the decay time, and the three short-period oscillations visible on the rising edge. To show this burst more adequately, we have plotted in Figure 2 the individual points of the digital record for April 3, 1967, over the time interval from 2200 to $3250 \mathrm{sec}$. This shows the unfiltered record, with photon statistics of 0.0065 mag per data point. The short-period fluctuations on the leading edge of the burst are clearly seen; this

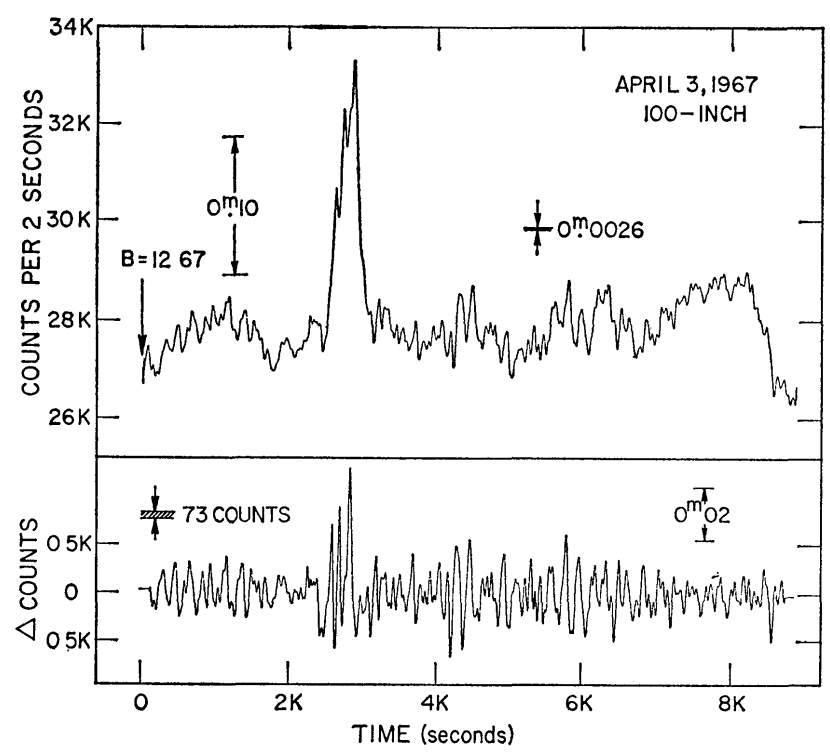

Fig. 1.-Upper: Computer-drawn digital record of the light curve for Sco X-1 on April 3, 1967. The original sky-subtracted data points, one for every 5-sec sampling interval, have been numerically smoothed with a Gaussian filter of 31-sec equivalent width. The effective photon statistics per smoothed data point is 0.0026 mag. The $B$ magnitude at the beginning is shown. Lower: The high-frequency component of the light fluctuations, obtained by subtraction as described in the text. An amplitude of 002 mag relative to the mean intensity level of the light curve is indicated.

shows that the high-frequency component persists during the flaring phase. As subsequently described, the conclusion is borne out from the records for the two subsequent nights when flaring occurred.

Figure 3 shows the computer-drawn digital record for 3.5 hours of continuous monitoring with the 200-inch reflector on April 13,1967. No flaring occurred on this night. The short-period fluctuations are present with a mean peak-to-peak amplitude of 0.015 mag. The gross feature of this record is an increase in the intensity from $B=13.32$ to $B=$ 12.85 , upon which a long-period wave of about 0.15 -mag amplitude with a characteristic time of about 1.5 hours is superposed. Non-coherent (unphasable) waves of this type are quite frequent in the data of Hiltner and Mook $(1967 a, b)$. Shown as an insert in Figure 3 is the record of continuous monitoring for 1.5 hours of a control star which had the same intensity as Sco X-1. The transparency on this night was comparable with the other four nights. We believe, therefore, that this forms an adequate control. The random variations over intervals of minutes is of the order of $0.004 \mathrm{mag}$, which is close to that due to photon statistics alone.

Perhaps the most interesting night of the five was that of May 12, 1967, when obser- 


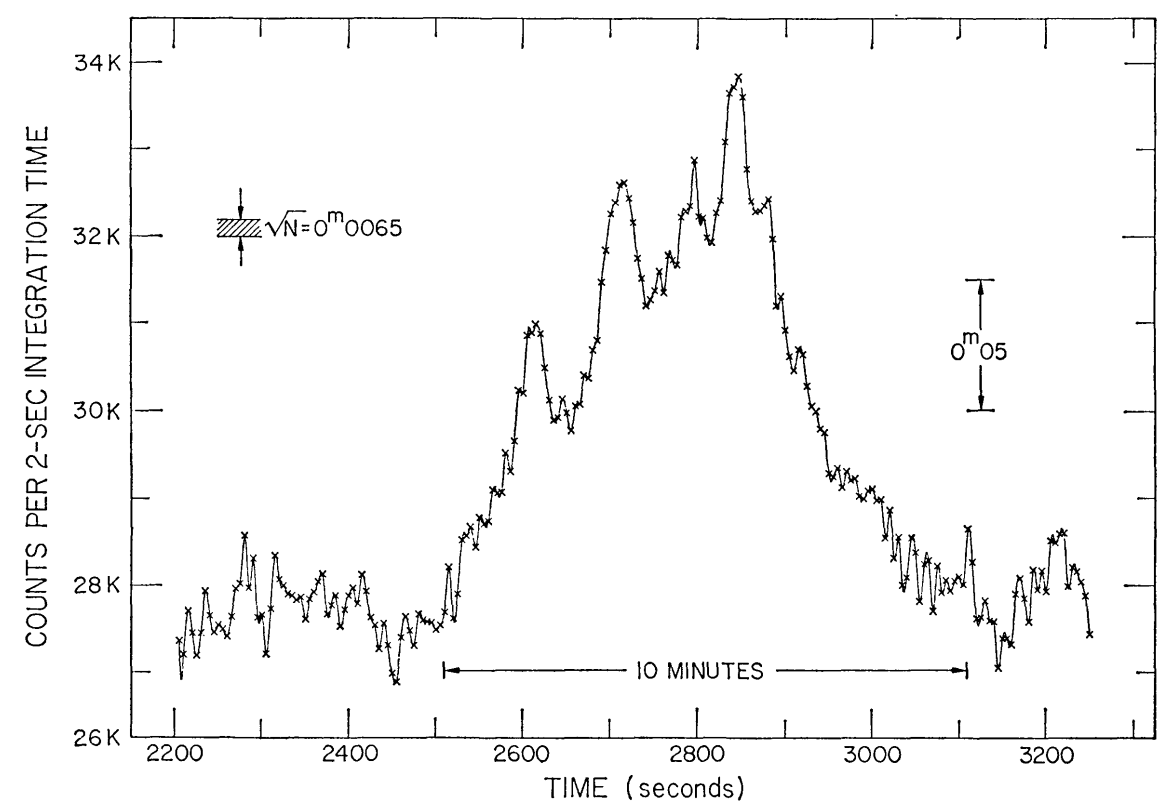

FIg. 2.-Portion of Fig. 1 in the 2200-3250-sec time interval. The original, unsmoothed, sky-subtracted data are plotted for each 5-sec sampling interval. The error due to photon statistics per data point is 0.0065 mag. A four-point interpolation curve has been drawn through the points by the computer.

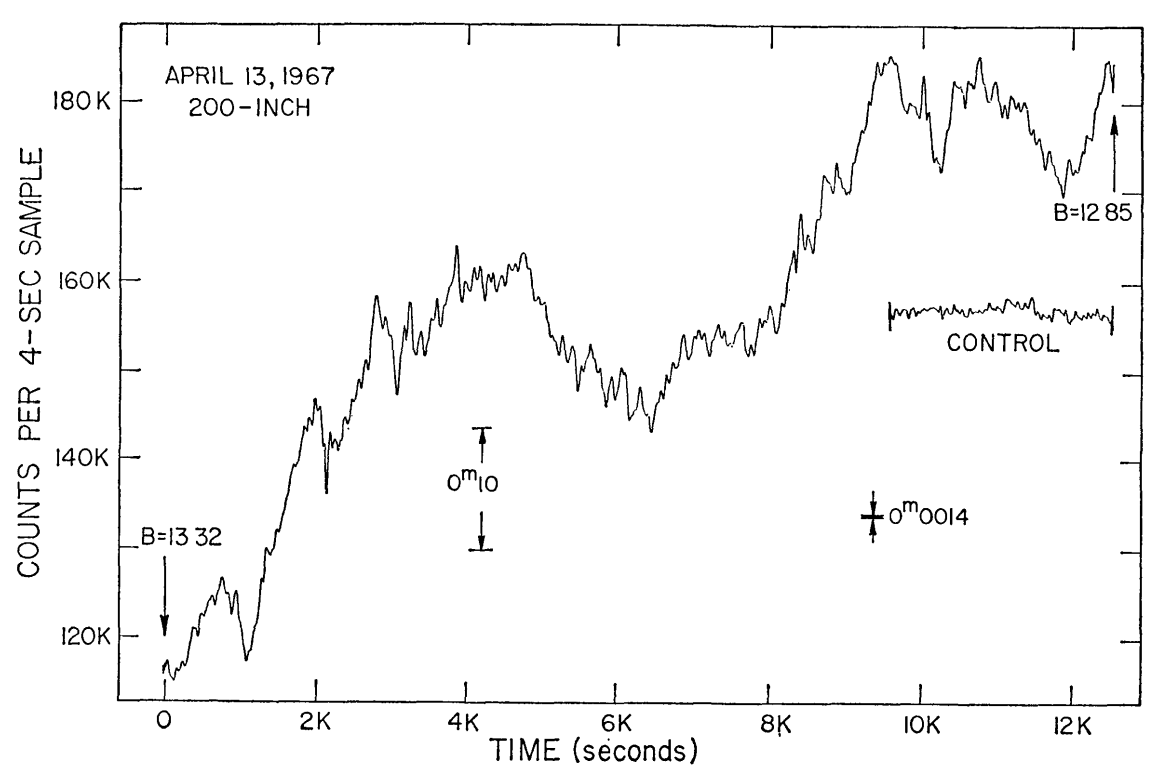

Frg. 3.-Light curve for April 13, 1967, obtained over a $3^{\mathrm{h}} 30^{\mathrm{m}}$ interval. As in Fig. 1, the data have been numerically smoothed with a Gaussian of 31-sec equivalent width, which gives an effective error per data point of 0.0014 mag due to photon statistics. A monitoring run of $1^{\mathrm{h}} 15^{\mathrm{m}}$ on a control star of the same intensity as Sco X-1 is shown. 
vations were made with the Mount Wilson 60-inch reflector using an S20 photomultiplier tube with no filter. Figure 4 shows that at least eight separate flares of amplitude $\sim 0.15$ mag occurred during the $4^{\mathrm{h}} 27^{\mathrm{m}}$ monitoring period. The high-frequency flickering was also present, as shown by the record at the bottom, which was separated from the complete light curve by the method already described.

The most obvious visual impression of the lower part of Figure 4 is that the amplitude of the flickering increases during the flaring activity, especially in the time interval from 7500 to 11500 sec. It is not clear, however, that this is real, because the subtraction procedure obviously breaks down in regions of rapid change of the total flux where the gradients of the light curve are steep. The problem is further complicated by the arbitrariness of definition of what constitutes the flickering and the flaring. For example, in the interval from 7500 to $11500 \mathrm{sec}$, the increased intensity at 8440 and $9690 \mathrm{sec}$ could

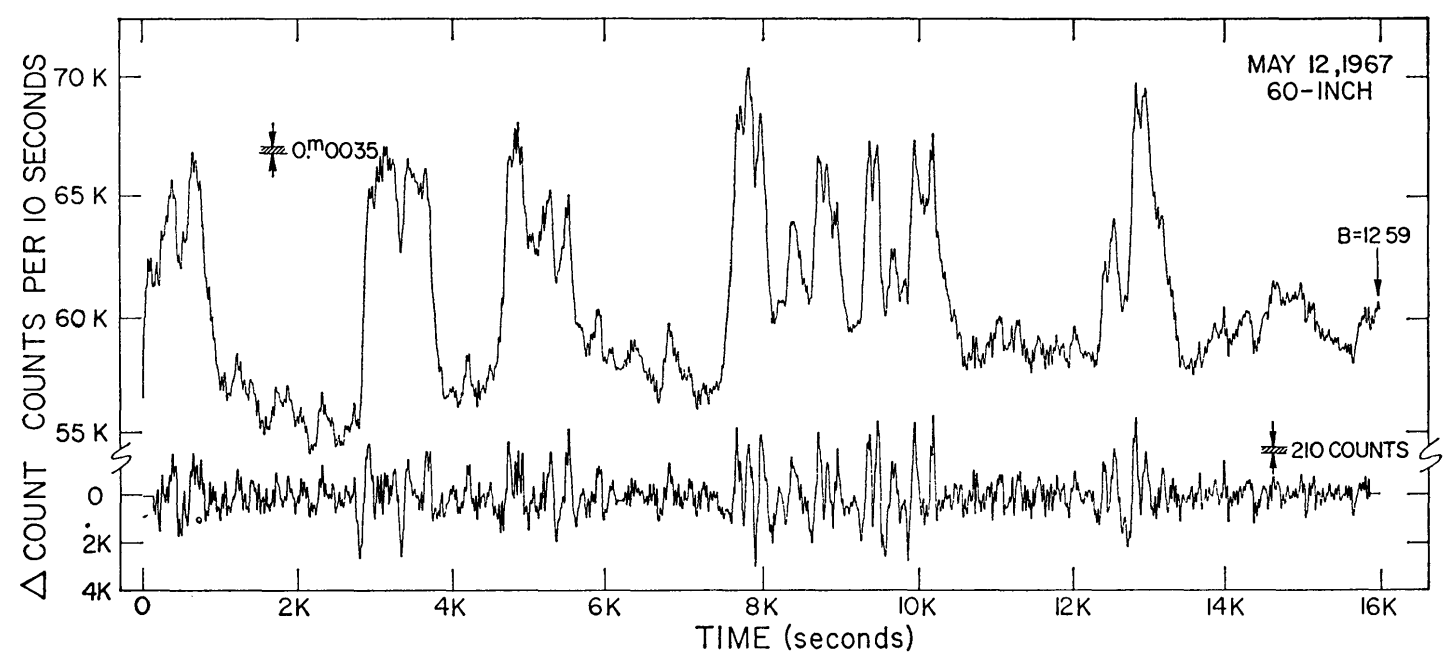

FIG. 4 - Light curve for May 12, 1967, obtained in $4^{\mathrm{h}} 27^{\mathrm{m}}$ with the 60 -inch telescope and numerically smoothed to give an effective error of 0.0035 mag per data point. The difference record is shown below.

be described as either minor flares or major flickers. Therefore, without knowledge of the underlying physical phenomenon, we can ascribe no significance to the increased flicker amplitude in those regions of major flares.

One of the remarkable features of Figure 4 is the steepness of the rise and decay of several of the flares. The most extreme example is the flare centered at time $3500 \mathrm{sec}$, where a rise of $0.16 \mathrm{mag}$ occurs in only $80 \mathrm{sec}$. The decay is almost as steep. The detailed digital record, with a four-point computer-drawn interpolation line superposed, is shown in Figure 5 for the interval between about 3000 and $6500 \mathrm{sec}$ of May 12. The original data have been numerically filtered with a 26 -sec Gaussian before plotting. With this filtering, the photon statistical error per data point is about 0.004 mag.

The data for the final two nights of monitoring are shown in Figure 6. Extensive flaring was observed on June 3,1967 . Sco X-1 was hardly quiescent during any of the $2^{\mathrm{h}} 45^{\mathrm{m}}$ of observation. No flaring was evident on June 5,1967 . On both nights the high-frequency flicker is obvious. The mean peak-to-peak amplitude of this activity was 0.016 mag on June 3, 1967, and only 0.009 mag on June 5, 1967.

Finally, Figure 7 shows the lightly filtered point-by-point digital record for the interval from 5000 to $8000 \mathrm{sec}$ on June 3,1967. This diagram shows particularly well the almost periodic fluctuations of 0.015 -mag amplitude on both the rising and falling branches of the flare. 


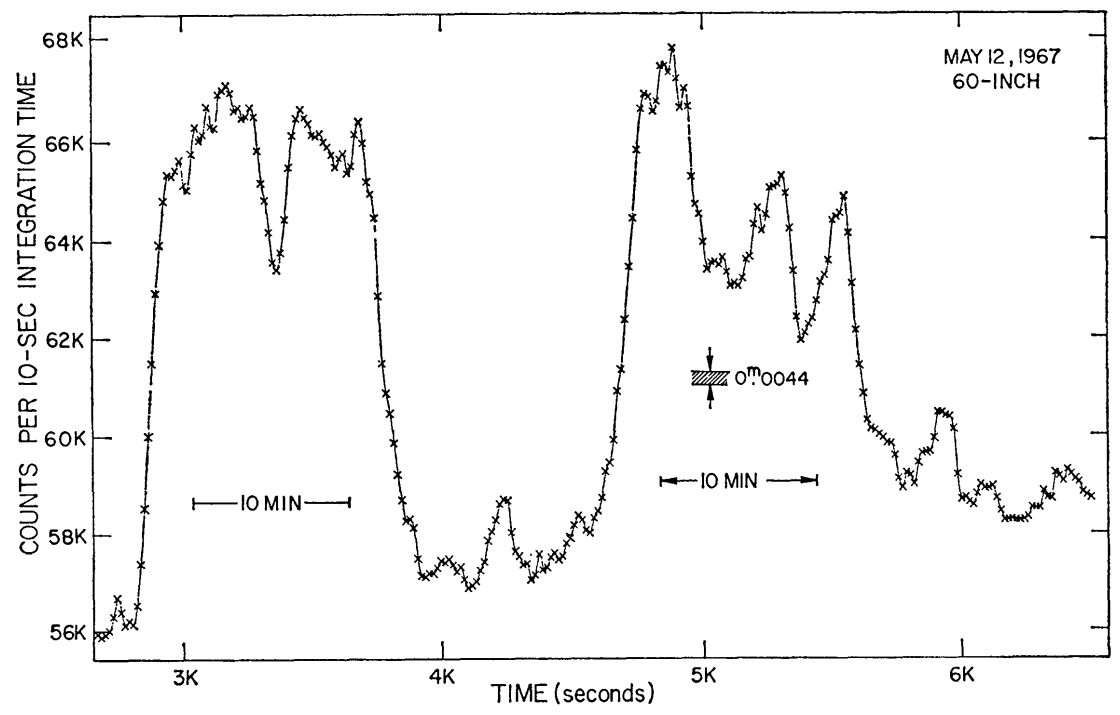

FIg. 5.-Portion of Fig. 4 between time markers at 2800 and $6400 \mathrm{sec}$, showing the individual data points with 15-sec sampling intervals. Record has been numerically smoothed with a Gaussian of 26-sec equivalent width, which gives an effective error of 0.0044 mag per data point due to photon statistics.

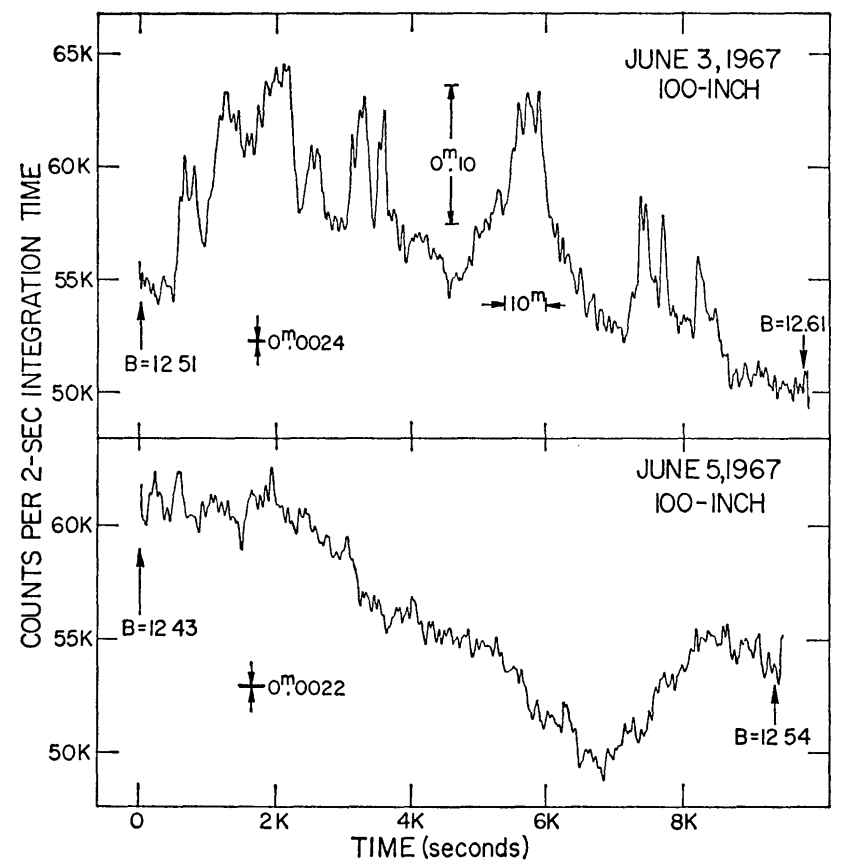

FIg. 6.-Light curves for June 3 and June 5, 1967, obtained with the 100-inch telescope and numerically smoothed with an equivalent 31-sec Gaussian filter. 
Complete plots of the data on the scale of Figures 2, 5, and 7, will be given in an Astrophysical Journal Supplement.

\section{RADIAL VELOCITIES}

The results set out in Figures 1-7 are generally similar to the fluctuations observed in Cyg X-2 (Kristian et al. 1967). They also resemble closely the short-term flickering observed in old novae and in nova-like variables of the $U$ Gem class, for which an extensive literature now exists. Variables with similar $U-B, B-V$ colors and with short-period flickering include (1) the old novae studied by Walker (1957) in his fundamental and now classic initial work, (2) MacRae $+43^{\circ} 1$ (Walker 1954), (3) SS Cyg (Grant 1955), (4) many stars observed by Mumford (1966a; see esp. Figs. 1 and 2), (5) DQ Her (Walker

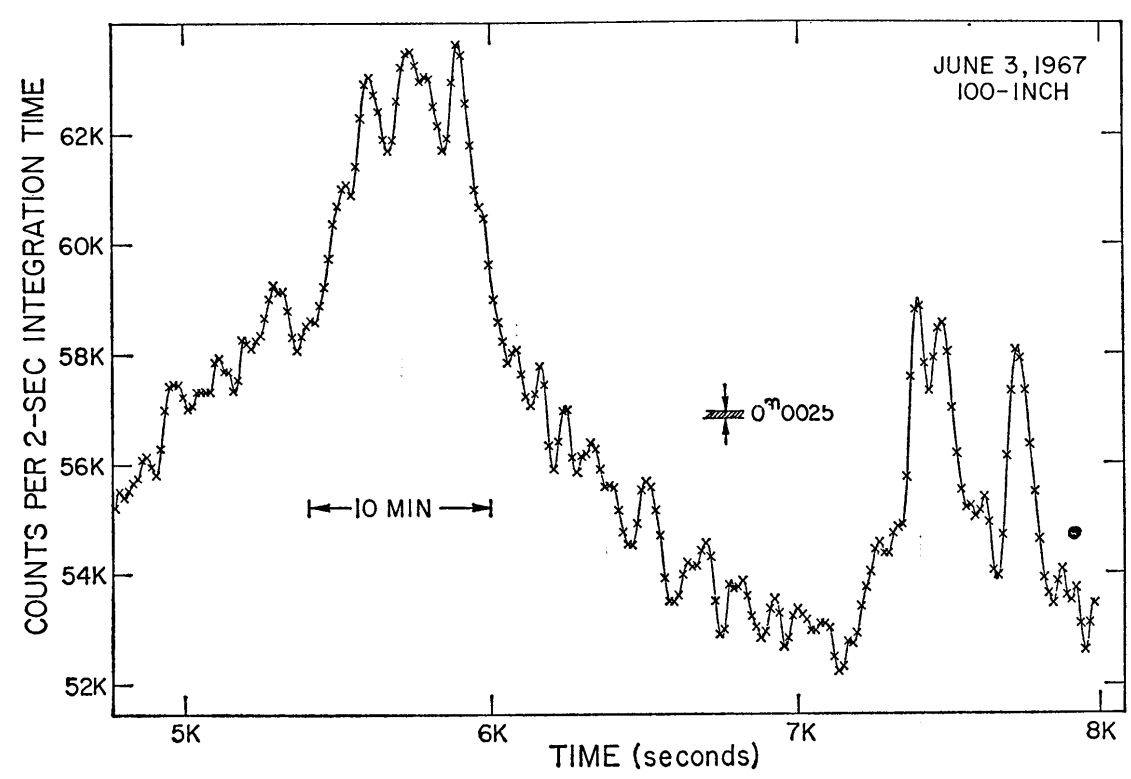

Fig. 7.-Portion of Fig. 6 for the light curve on June 3, 1967, between time markers at 5000 sec and $8000 \mathrm{sec}$, smoothed with a 26-sec Gaussian filter to give an effective statistical error of 0.0025 mag per data point. Only every third original 5 -sec data point is plotted.

1956, 1958, 1961), (6) U Gem (cf. Mumford 1964; Krzeminski 1965), (7) RW Tri (Walker 1963a), (8) T Aur (Walker 1963b), (9) WZ Sge (Krzeminski 1962), and others.

In view of this similarity of photometric properties and in view of the binary nature of Cyg X-2 (Burbidge, Lynds, and Stockton 1967; Kristian et al. 1967), a property shared by all old novae and U Gem variables (Joy 1956; Kraft 1962, 1964), the question arises whether Sco X-1 is a binary. Visual inspection of the spectra now available (Sandage et al. 1966) failed to show any large variation or radial velocity, and, in particular, revealed no large S-wave distortion of the lines on the singly trailed plates. However, unpublished measurements of the plates by Jugaku (Babcock 1967) and by Osmer suggest that radial-velocity variations are present, although the changes are near the limit of conventional detectability. Because of the importance of the question, we have remeasured the plates by a digital method (Westphal 1966) that appears to give considerably higher accuracy.

All available spectrograms were traced with a microphotometer, whose photoelectric output was amplified and sent by underground cable to the California Institute of Technology computing center. There the signal was sampled every $3.8 \mu(0.3 \AA)$ and 
stored in digital form on magnetic tape by a high-speed analogue-to-digital converter. The projected microphotometer slit width $(0.5 \AA)$ and the sampling interval $(0.3 \AA)$ are both well below the resolution limit of the plates $(\sim 20 \mu$ or $1.7 \AA)$, which means that all real information was recorded.

The singly trailed plates were microphotometered in successive segments, each equivalent to about 10-min elapsed time at the telescope, to look for variations over the trailing intervals.

To measure velocities, the digital data for each microphotometer run were numerically smoothed by an appropriate Gaussian filter using the procedure previously described (Westphal 1966). The centers of the emission lines were located as positions of zero slope to within $\pm 0.1 \AA$ relative to the comparison lines, which had been reduced by the same method. Detailed study of the slit-curvature correction, which is appreciable with the wide spectra, was made from a plate exposed to the helium comparison source over the full length of the long slit. The correction, determined by tracing and analyzing many small segments of the test plate, was applied by computer to each of the data runs on Sco X-1. The correction was checked by noting the wavelength constancy of the contaminating San Diego mercury lines (mislabeled as Los Angeles lines in the reproductions of Sandage et al. 1966). No systematic variation of the corrected $\lambda(\mathrm{Hg})$ across the plates was present at the $\pm 0.8 \AA\left(10 \mu\right.$ on the $85 \AA \mathrm{mm}^{-1}$ plates $)$ level.

The three strongest lines, $\mathrm{He}$ II $(\lambda 4686), \mathrm{H} \gamma$, and $\mathrm{H} \delta$, were measured for radial velocity on all available plates. The errors, estimated to be somewhat less than $\pm 50 \mathrm{~km}$ $\sec ^{-1}$ ( $8 \mu$ on the plate), arise mainly from properties of the plate rather than from measurement or reduction procedures.

Analysis of the data for the two best single-trailed plates, N 2868 and N 2870, taken on July 17,1967 , and July 18, 1967, reveals velocity changes over a 3-hour interval, with the $\mathrm{He} \mathrm{II}$ and the hydrogen lines moving oppositely. In the time covered by $\mathrm{N} 2868$, $\mathrm{He}$ II varies monotonically from -155 to $-215 \mathrm{~km} \mathrm{sec}^{-1}, \mathrm{H} \gamma$ from -125 to $-35 \mathrm{~km}$ $\mathrm{sec}^{-1}$, and $\mathrm{H} \delta$ from -192 to $-112 \mathrm{~km} \mathrm{sec}^{-1}$. The change can be seen on visual inspection of the reproduction given elsewhere (Sandage et al. 1966, Fig. 4), by comparison of $\mathrm{H \gamma}$ with the adjacent mercury nightsky lines at $4358 \AA$. A similar variation over a 3 -hour run occurs on $\mathrm{N} 2870$ taken one night later. Here He II varies from -155 to $-270 \mathrm{~km}$ $\mathrm{sec}^{-1}, \mathrm{H} \gamma$ (with low accuracy) from -160 to $-90 \mathrm{~km} \mathrm{sec}^{-1}$, and $\mathrm{H} \delta$ from -250 to -135 $\mathrm{km} \mathrm{sec}^{-1}$. We emphasize again that on both nights the time variation of helium mirrors that of hydrogen.

Additional evidence exists for variations of velocity on this short time scale of several hours. (1) Two other singly trailed exposures of lower quality, N 2865 and N 3237, are available. On these, the $\mathrm{He} \mathrm{Ir}$ line does appear to change during the trailing interval of about $2 \frac{1}{2}$ hours but at a smaller rate than for the two high-quality plates. The hydrogen lines, although more uncertain, also probably vary. (2) On the first three nights of observation, July 13-15, 1966, two short-exposure plates were taken on each night, separated in time by about 2 hours. Table 2 lists the velocities measured on each of the two plates on each of the three nights. It is evident that significant changes occurred in the velocity of $\mathrm{H} \gamma$ and $\mathrm{H} \delta$ over a 2 -hour period, while the $\mathrm{He}$ Ir lines remained sensibly constant within a given night, except perhaps for July 15, 1966, where it may have changed by $60 \pm 50$ $\mathrm{km} \mathrm{sec}^{-1}$.

In addition to velocity changes within a given night, there is evidence for changes from night to night. Table 2 lists the mean velocities for each of the ten available plates. Several points should be noted. (1) The velocities of $H \delta$ are systematically smaller than those of $\mathrm{H} \gamma$ by $60 \pm 30$ (m.e.) $\mathrm{km} \mathrm{sec}^{-1}$. The rest of the Balmer lines, while less accurate, are consistent with $\mathrm{H} \gamma$, which suggests that the velocities from $\mathrm{H} \delta$ are anomalously low. The effect can be explained by contamination of $\mathrm{H} \delta$ by the expected high-excitation $\mathrm{N}$ III lines at 4097.3 and $4103.4 \AA$ that must be present if the identification of the $\mathrm{N}$ III 
complex near $4460 \AA$ is correct (Sandage et al. 1966, Fig. 4). Although these lines bracket $\mathrm{H} \delta$ at $4101.7 \AA$, there will be a bias toward shorter wavelengths, and the velocity of the combination should be more negative than from $\mathrm{H} \delta$ alone. With this in mind, from our measurements it seems likely that the velocities of all hydrogen lines are the same on any given plate. (2) Over the 6-day interval July 13-18, 1966, He II shows a systematic decline from -80 to $-200 \mathrm{~km} \mathrm{sec}^{-1}$. During the same interval, the hydrogen lines perhaps show, although with large scatter, a trend in the opposite direction.

The interpretation of the systematic trend of $\mathrm{He}$ Ir over a period of days is not obvious in view of the equally large velocity variation seen in an interval of hours. If the velocity change within a single night were due to simple binary motion, the indicated period would be less than one day, and a systematic variation over six days would not be expected if only two stars are involved. This anomaly would suggest that simple binary

TABLE 2

RADIAL VELOCITIES OF SCO X-1

\begin{tabular}{c|c|c|c|c|c}
\hline \hline Date (U T ) & Plate No. & $\begin{array}{c}\text { He Ir 4686 } \\
\left(\mathrm{km} \mathrm{sec}^{-1}\right)\end{array}$ & $\begin{array}{c}\mathrm{H} \gamma \\
\left(\mathrm{km} \mathrm{sec}^{-1}\right)\end{array}$ & $\begin{array}{c}\mathrm{H} \delta^{*} \\
\left(\mathrm{~km} \mathrm{sec}^{-1}\right)\end{array}$ & $B$ mag \\
\hline 1966: & N 2849 & -98 & -53 & -46 &. \\
July 13 23 & N 2851 & -98 & -164 & -221 & $\ldots$ \\
July 13 35 & N 2854 & -128 & -157 & -265 & 132 \\
July 14 19 & N 2856 & -128 & -74 & -171 & 13 \\
July 14 34 & N 2859 & -200 & -143 & -243 & 1285 \\
July 15 21 & N 2861 & -141 & -95 & -112 & 1260 \\
July 15 31 & N 2865 & -162 & -60 & -119 & 1265 \\
July 16 29 & N 2868 & -181 & -85 & -155 & 1345 \\
July 17 28 & N 2870 & -213 & -157 & -199 & 1260 \\
July 18 24 & N 3237 & -181 & & -265 & \\
July 31.24 & & & & \\
\hline
\end{tabular}

* $\mathrm{H} \delta$ velocities appear to be too negative by $60 \pm 30$ (m e.) $\mathrm{km} \mathrm{sec}^{-1}$ relative to $\mathrm{H} \gamma$ and all other Balmer lines, due possibly to contamination from $\mathrm{N}$ III ( $(\lambda \lambda 4097$ and 4103$)$.

motion cannot explain the observations, although the mirror behavior of the hydrogen and helium lines over 3-hour intervals on two nights would so suggest. However, we hesitate to suggest that more than two stars are involved. The possibility exists that the observed velocity changes could be due to gas streams rather than to orbital motions.

A minimum statement is that some systematic motion is present within the system, and in this respect Sco X-I is similar to the binary X-ray source Cyg X-2 (Burbidge et al. 1967; Kristian et al. 1967). Our data are not sufficient to settle this question.

\section{NEW SPECTROSCOPIC FEATURES}

During the analysis of the filtered microphotometer data just described, we saw clear indication of many faint emission lines which are only marginally visible on the original spectrograms. All the weak, broad lines marked on the reproductions given elsewhere (Sandage et al. 1966, Fig. 4) show clearly on the digital records. We have not made an exhaustive study to detect and list all the lines present but have carried the analysis to such a point that we are convinced of the power of this digital method for weak-line recovery.

Individual strips of those plates which had been singly traced for the time sequences were added by computer to produce a record equivalent to that which would have been 
obtained by a long microphotometer slit covering the entire plate. The digital record for each plate was then filtered sufficiently to remove much of the remaining plate noise. A smoothed mean continuum level was then subtracted by computer from each record. The difference records, which now represent real spectral lines plus residual noise, were then compared to search for features common to all plates.

All the prominent lines previously noted (Sandage et al. 1966) were recovered. The strongest line, visible on all plates, is $\mathrm{He}$ II $(\lambda 4686)$. The broad diffuse band centered at $4640 \AA$, suggested to be $\mathrm{N}$ III with possible $\mathrm{C}$ III and $\mathrm{O}$ II contributions, is partially resolved into at least four narrow lines on some of the plates, but on others the band is structureless. The resolution is particularly well shown on the plate N 2865 for July 16.29, which is the top reproduction in Figure 4 of Sandage et al. (1966). Real changes occurred in the diffuseness of this feature over the six-night interval, changes which may be similar in certain aspects to nitrogen flaring commonly observed in novae at particular times. Furthermore, the presence of the $4640 \AA$ feature, which is relatively rare in stellar spectra, is characteristic of most novae at a phase starting about 3.5 mag below maximum light after an outburst (Payne-Gaposchkin 1957, pp. 296 ff.; McLaughlin 1960).

All the Balmer-series lines are present to at least H12. We have found numerous lines of He I, among which are $\lambda \lambda 3634,3819,4026,4121,4144,4169,4472$, and 4713. Lines of He II were detected at $\lambda \lambda 3858$ and 4541 . The prominent $\mathrm{O}$ II $(\lambda 4415)$ line was found on all ten plates.

The spectrum is filled with weak, broad features between 4350 and $4600 \AA$, some of which were previously marked in the published reproduction as due to $\mathrm{N}$ III, He I, $\mathrm{O}$ Ir. All of these show well on the microphotometer records, typical examples of which are shown in Figure 8 (Plate 2). The complete tracing shown is of plate N 2854 taken at a time when the hydrogen lines were strong relative to the continuum. The inserted segment in Figure 8 is for plate N 2870, when Sco X-1 was bright $(B=12.6)$. The hydrogen lines are very weak relative to the continuum on this plate, but the faint features in the interval 4350-4600 $\AA$ show well.

Various identified emission lines are marked in Figure 8 (Plate 2). The parentheses inclose the number of plates on which each line was detected. In addition to those features marked, we found consistent evidence from plate to plate for lines whose identification is unknown. The lines found on six or more plates include $\lambda \lambda 3944$ (8 plates), 3990 (7), 4295 (6), 4318 (8), 4374 (6), 4602 (8), and 4731 (6). The lists of emission lines in the high-excitation planetary nebula NGC 7027 (Aller, Bowen, and Minkowski 1955; Aller, Bowen, and Wilson 1963), in the Orion Nebula (Kaler, Aller, and Bowen 1965), and in the recurring nova RS Oph (Joy and Swings 1945; Joy 1961) were searched for identification, but with no positive results. Possibly our line at $4374 \AA$ is $N$ III, whose laboratory wavelength is $4379 \AA$. No convincing identifications exist for forbidden lines, but it should be emphasized that we have not thoroughly studied the identification problem.

The well-observed line at $4232 \AA$ (7 plates) may be Fe II because other expected lines from this ion near 4254 (5 plates), 4489 (5), 4543 (7), and $4551 \AA$ (6) were detected. However, acceptance of this identification should be reserved until the yellow spectral region is searched for the strong lines at 5197, 5234, 5275, and $5313 \AA$ due to Fe II.

\section{SPECTRAL CHANGES}

The most impressive single characteristic of the spectroscopic data is the large intensity variation of the hydrogen lines from night to night, relative to the continuum. On those nights when Sco X-1 was bright in the broad-band $U B V$ system, the hydrogen lines were very weak, and conversely. The same comment applies to lines of He I, es- 
pecially $\lambda 4472$, and $\lambda 3889$ which is blended with H8. The direction of the apparent changes is to keep the absolute intensity of the hydrogen lines constant as the continuum varies.

Unfortunately, none of the 1966 spectra was calibrated, and accurate equivalent widths cannot be measured. However, using a calibration wedge taken with the Sco X-1 plate $\mathrm{N} 3237$ on July 31, 1967, we have measured approximate equivalent widths for the $\mathrm{N}$ III complex at $4640 \AA$, for $\mathrm{He}$ II $(\lambda 4686), \mathrm{H} \gamma$, and $\mathrm{H} \delta$. The result, to within errors of \pm 20 per cent, is that the lines remain constant in absolute flux as the continuum varies. A reasonable conclusion from this result is that the continuum flux and the line emission are decoupled-they arise in different regions of Sco X-1 under different physical conditions. The existence of regions of high and low excitations is also evident in the simultaneous appearance of low-excitation lines $(\mathrm{H}, \mathrm{He} \mathrm{I})$ and high-excitation lines ( $\mathrm{N}$ III, O III, He II) in the same spectrum. It is not clear from our data how the intensities of the faint, broad bands between 4350 and $4600 \AA$ change. We have some indication that these features do not vary relative to the continuum as the magnitude of Sco X-1 changes but rather that the absolute intensity keeps step with the continuum flux. The point must obviously be checked because, if real, these lines may be formed within the same environment and may be controlled by the same processes as the continuum.

\section{DISTANCE TO SCO $\mathrm{X}-1$}

To determine the total X-ray power of Sco X-1 requires knowledge of the distance. There appear to be three independent methods of varying precision and reliability by which limits can be set.

\section{a) Strength of Interstellar $\mathrm{Ca}$ II K}

The K-line of Ca II is clearly visible on each of the $85 \AA \mathrm{mm}^{-1}$ spectrograms. The $\mathrm{Ca}$ II $\mathrm{H}$-line is blended with $\mathrm{H} \epsilon$ in emission and is visible only on nights when $\mathrm{H} \epsilon$ effectively disappears, when Sco X-1 is bright. These lines are most probably interstellar, rather than circumstellar as in Cyg X-2 (Kristian et al. 1967), because the mean velocity of $\mathrm{Ca}$ II $\mathrm{K}$ from all ten plates is $-1 \pm 15 \mathrm{~km} \mathrm{sec}^{-1}$, which differs from any value in Table 2. Furthermore, Wallerstein's (1967) more accurate value of $-6.8 \mathrm{~km} \mathrm{sec}-1$ agrees with interstellar cloud velocities for five stars in the direction of Sco X-1.

The equivalent width of $\mathrm{Ca}$ II $\mathrm{K}$ is estimated from our plate to be $250 \mathrm{~m} \AA$, which agrees with Wallerstein's measured value of $280 \mathrm{~m} \AA$. There are no known stars within $300 \mathrm{pc}$ whose interstellar Ca II line is this strong. The correlation between distance and $\mathrm{K}$-line strength averaged over the entire galactic plane, derived by Beals and Oke (1953), shows that $D$ (Sco X-1) $\geq 500$ pc. The correlations of Binnendijk (1952; cf. Figs. 5 and 6) for the regions near Sco X-1 suggest $D$ (Sco X-1) $\simeq 600 \mathrm{pc}$. However, these statistical relations are only suggestive, since they do not preclude chance occurrence of heavy clouds in front of particular objects.

Wallerstein's (1967) investigation of the line strengths for stars close to the direction of Sco X-1 places a firm lower limit of $D$ (Sco X-1) $\geq 270$ pc. The distance to Sco X-1 can of course be considerably larger than these lower-limit values because at $b=+24^{\circ}$ the line of sight passes out of the galactic plane $(Z=150 \mathrm{pc})$ for $D>370 \mathrm{pc}$, and the strength of Ca II $\mathrm{K}$ should not appreciably increase if Sco X-1 were put at any place beyond this point. We note in passing that these results suggest that Sco X-1 is not a member of the Scorpio-Centaurus association (Braes and Hovenier 1966) at $D \simeq 170 \mathrm{pc}$ (Bertiau 1958) because the strength of $\mathrm{Ca}$ II $\mathrm{K}$ in all member stars is considerably less than $280 \mathrm{~m} \AA$ (Binnendijk 1952, Table 5).

The preceding arguments depend, of course, on assuming that the K-line absorption 
is entirely interstellar. If part or all of it is associated in some unknown way with Sco $\mathrm{X}-1$ itself, notwithstanding the radial-velocity agreement with interstellar cloud velocities in this direction, then this discussion is vitiated. With no known reason for this assumption, we accept $D \geq 270 \mathrm{pc}$ from the K-line strength as a very firm lower limit to the distance.

\section{b) Number of Hydrogen Atoms in Line of Sight}

A less-certain estimate is available by calculating the number of hydrogen atoms in the line of sight, following the method of Münch (1966). The observed doublet ratio $\mathrm{K} / \mathrm{H}=1.4$ (Wallerstein 1967) permits an estimate of the number of calcium atoms in the line of sight, $N_{\mathrm{Ca} \text { n }} D$ to Sco X-1. If we enter the curve of growth for a Gaussian velocity distribution of the interstellar clouds with equivalent width $(\mathrm{H})=200 \mathrm{~m} \AA$ and $\mathrm{K} / \mathrm{H} \simeq 1.4$, we obtain $N_{\mathrm{Ca} \text { II }} D=6 \times 10^{12} \mathrm{~cm}^{-2}$ (Münch 1958, Fig. 6) with an uncertainty of less than a factor of 2. To this accuracy the result is not sensitive to the doublet ratio, which cannot be accurately determined in Sco X-1.

The number of hydrogen atoms in the line of sight can then be found by assuming that the $\mathrm{K}$-line is formed entirely in $\mathrm{H} \mathrm{I}$ regions and that cosmic abundance ratios of calcium and carbon (which supply all the electrons) to hydrogen apply (cf. Münch 1966). We adopt the radiation field as defined by Münch 1966) and take the abundance ratios as $N(\mathrm{H}) / N(\mathrm{Ca})=6 \times 10^{5}$ and $N(\mathrm{H}) / N(\mathrm{C})=3 \times 10^{3}$ (Aller 1961, Table 8-3) and get, from the equation of ionization equilibrium,

$$
N_{\mathrm{H}^{2}}{ }^{2}=2.7 \times 10^{20} \mathrm{~cm}^{-5},
$$

where $N_{\mathrm{H}}$ is the number of $\mathrm{H}$ atoms per cubic centimeter and $D$ is the path length in centimeters.

Studies of $21-\mathrm{cm}$ radiation show that the average value for $N_{\mathrm{H}}$ in the plane is $0.5 \pm$ 0.1 atom $\mathrm{cm}^{-3}$ (cf. Kerr and Westerhout 1964). The hydrogen is concentrated close to the plane with a height of 110 pc to the half-density point (Schmidt 1957). This means that at latitude $b=+24^{\circ}$ the average $N_{\mathrm{H}}$ over the entire line of sight will be $N_{\mathrm{H}} \simeq 0.4$ $\mathrm{cm}^{-3}$ for distances $D \simeq 400 \mathrm{pc}(Z \simeq 100 \mathrm{pc})$. Combining this with our value of $N_{\mathrm{H}}{ }^{2} D$ gives $D$ (Sco $\mathrm{X}-1) \geq 240 \mathrm{pc}$.

The result is a lower limit unless we can show that Sco X-1 is still within the galactic layer of interstellar absorption. That is, if there is more matter beyond Sco X-1, then moving it to a greater distance would increase the observed absorption, but if $N_{\mathrm{H}}{ }^{2} L=$ $2.7 \times 10^{20} \mathrm{~cm}^{-5}$ is the galactic limiting value in this direction, Sco X-1 could be at any greater distance $D>L$. On this point, McGee and Murray (1961, Fig. 1) show that the total hydrogen path length through the Galaxy in the direction of Sco X-1 is $N_{\mathrm{H}} L=$ $1.8 \times 10^{21} \mathrm{~cm}^{-2}$. Taking our value of $N_{\mathrm{H}}^{2} D=2.7 \times 10^{20}$ up to Sco X-1 itself, and adopting $\left\langle N_{\mathrm{H}}\right\rangle=0.4$, gives the path length to Sco X-1 as $N_{\mathrm{H}} D=6.7 \times 10^{20}$, which is about three times smaller than the total path length to outside the Galaxy. If true, then $D=$ $240 \mathrm{pc}$ is not a lower limit but an actual determination of the distance itself. It should be noted, however, that although the argument appears to be logically consistent, the uncertainties are rather large.

\section{c) X-Ray Optical Depth}

For values of $N_{\mathrm{H}} D$ near $10^{21} \mathrm{~cm}^{-2}$, interstellar absorption of soft $\mathrm{X}$-rays with energies less than $2 \mathrm{keV}$ is expected. The absorption coefficient per hydrogen atom of the interstellar gas with normal cosmic abundances is $\sigma(1 \mathrm{keV})=4 \times 10^{-22} \mathrm{~cm}^{2}$ (Felton and Gould 1966), which gives $\tau(1 \mathrm{keV})=0.4$ for the stated path length. Unfortunately, the available X-ray data are insufficient for a firm answer on $\tau$ (Sco X-1) near $1 \mathrm{keV}$. 
Spectra by Grader et al. (1966) suggest that $\tau(1 \mathrm{keV}) \simeq 0.2$ (with a large error), which requires $N_{\mathrm{H}} D=5 \times 10^{20} \mathrm{~cm}^{-2}$, or $D=380 \mathrm{pc}$. However, the result is so sensitive to the value of $\tau$, which is experimentally difficult to determine at $1 \mathrm{keV}$, that the result has low weight. Gorenstein, Giacconi, and Gursky (1967) report a formal value of $\tau \simeq 1$ at $1 \mathrm{keV}$, but they consider their result uncertain enough to permit a conclusion of zero absorption $(\tau=0)$ at this energy, a result which is consistent with another experiment by the American Science and Engineering group, reported by Gorenstein, Gursky, and Garmine (1968). If $\tau(1 \mathrm{keV})=1$ is taken as an upper limit, then $N_{\mathrm{H}} L<2.5 \times 10^{21} \mathrm{~cm}^{2}$, which, in view of $\S \mathrm{VI} b$, is not an interesting number. If, however, the conclusion of Gorenstein et al. (1967) holds that $\tau<1$, then the conclusions of $\S \mathrm{VI} a$ and $b$ are reinforced.

\section{d) Sco X-1 as an Old Nova}

The optical characteristics of Sco X-1 resemble old novae and U Gem variables in the following ways: (1) similar and rare $U B V$ colors, (2) similar optical spectra, (3) large radial-velocity changes in periods of several hours suggestive of binary motion, and (4) rapid flickering and minor outbursts. The similarities are so striking that we provisionally adopt Sco X-1 as an old nova and obtain thereby an independent estimate of distance.

TABLE 3

\section{SummaRy of DISTANCE Estimates}
Method
a) K-line intensity .
$>270$ pc (Wallerstein 1967)
$\geq 500 \mathrm{pc}$ (Beals and Oke 1953)
$\simeq 600 \mathrm{pc}$ (Binnendijk 1952)
b) Hydrogen path length
$=240 \mathrm{pc} ; N_{\mathrm{H}^{2} L}$ with $N_{\mathrm{H}}=0.4$
$=425 \mathrm{pc} ; N_{\mathrm{H}}{ }^{2} L$ with $N_{\mathrm{H}}=0.3$
c) X-ray optical depth
$\simeq 400 \mathrm{pc}$ if $\tau(1 \mathrm{keV})=0.2$
d) Old nova......
$\leq 2000 \mathrm{pc}$ if $\tau(1 \mathrm{keV})<1$
$\leq 2000 \mathrm{pc}$ if $\tau(1 \mathrm{ke})$
$230<D<1000 \mathrm{pc}$

At minimum light, $M_{B}$ for old novae spread from +2 to +7 absolute magnitude (Payne-Gaposchkin 1957, Table 1.16). We adopt $\langle B\rangle_{0}=12.1$ for Sco X-1, obtained from the observed $\langle B\rangle \simeq 13.0$ with $A_{B}=4 E(B-V) \simeq 0.9 \mathrm{mag}$, and get $230<D<$ 1000 pc. However, this lower limit of 230 pc puts Sco X-1 unacceptably close, based on the strength of the $\mathrm{K}$-line. On the other hand, the possible range of $M_{B}$ permits $(m-M)_{0}$ $=10.1$, or $D=1000 \mathrm{pc}$, as an upper limit. A realistic range of distance on this basis is then $1000>D>300$ pc.

\section{e) Summary}

Table 3 summarizes the distance estimates. A firm conclusion from method $a$ is that $D$ is surely greater than $300 \mathrm{pc}$. Method $b$ suggests that Sco X-1 is not beyond the galactic total thickness in $\mathrm{H}$, while method $c$ shows that $D=1000 \mathrm{pc}$ is the upper limit if Sco X-1 is like the brightest known old nova at minimum light. To within the respective uncertainties, the total material is consistent with $\langle D\rangle \simeq 500 \mathrm{pc}$, which is adopted.

\section{RADIATED X-RAY POWER, COOLING TIME, AND THE OPTICAL FLUCTUATIONS}

The shape and absolute calibration of the $\mathrm{X}$-ray spectrum from 1 to $20 \mathrm{keV}$ have been determined by several experimenters using at least seven different rocket flights. There is general agreement in the absolute calibration within a factor of about 2. To 
within this accuracy, the observed spectrum can be approximated over the stated energy range by

$$
S=100 e^{-E / 40} \mathrm{keV} \mathrm{keV}^{-1} \mathrm{~cm}^{-2} \mathrm{sec}^{-1},
$$

where $E$ is the energy expressed in kiloelectron volts (for a summary cf. Grader et al. 1966; Gorenstein et al. 1968). Conversion to more familiar units gives

$$
S=6.6 \times 10^{-25} e^{-E(\mathrm{keV}) / 40} \mathrm{erg} \mathrm{sec}^{-1} \mathrm{~cm}^{-2} \mathrm{~Hz}^{-1},
$$

where the $e$-folding energy of $4.0 \mathrm{keV}$ corresponds to a temperature of $4.6 \times 10^{7} \mathrm{~K}$. By integrating the monochromatic flux over all frequencies, we obtain a total flux at the Earth's surface of

$$
F=6.4 \times 10^{-7} \mathrm{erg} \mathrm{cm}^{-2} \mathrm{sec}^{-1},
$$

which, with $D=500 h \mathrm{pc}$, requires a radiated power of

$$
P=2 \times 10^{37} h^{2} \text { erg } \sec ^{-1} \text {. }
$$

Here $h$ is the distance in units of $500 \mathrm{pc}$.

As pointed out by many people (Johnson 1966; Burbidge 1967; Rossi 1968), the assumption of a homogeneous bremsstrahlung source allows the calculations of many of the physical properties of a model. Adopting the volume emissivity of optically thin bremsstrahlung, integrated over all frequencies (cf. Spitzer 1949; Greene 1959; Elwert 1961; Tucker and Gould 1966), as

$$
\epsilon \simeq 1.5 \times 10^{-27} T^{1 / 2} N_{e}^{2} \bar{g} \operatorname{erg~} \mathrm{cm}^{-3} \mathrm{sec}^{-1},
$$

and assuming a spherical model of radius $R$, we arrive at a total power of $4 / 3 \pi R^{3} \epsilon$ which, when equated to the observed power of equation (4) with $T=5 \times 10^{7} \mathrm{~K}$ and $\bar{g}=1$, gives

$$
\left\langle N_{e}^{2}\right\rangle^{1 / 2}=6.6 \times 10^{29} h R^{-3 / 2} \mathrm{~cm}^{-3} .
$$

The total energy store of this gas is $\sim 3 k T\left\langle N_{e}^{2}\right\rangle^{1 / 2} \frac{4}{3} \pi R^{3}$ which, when divided by the radiated power (eq. [4]), gives the cooling time of

$$
t_{c}=3 \times 10^{-15} h^{-1} R^{3 / 2} \mathrm{sec} .
$$

From the evidence of Cyg X-2 where a separation of the binary stars of the order of 0.01 a.u. is suggested (Kristian et al. 1967) and from an apparently similar situation in Sco X-1, we assume that $R$ lies in the range from $10^{11}$ to $10^{12} \mathrm{~cm}$, which requires $t_{c}$ to be in the range $100 h^{-1}<t_{c}<3000 h^{-1}$ sec.

The importance of this number is that it sets the time scale for energy input into the system. To maintain its X-ray output over days, Sco X-1 must renew the entire energy supply of its gas every $t_{c}$ seconds. We are forced to conclude that Sco X-1 must continuously pump itself on the scale of minutes at an average rate of $2 \times 10^{37} h^{2} \mathrm{erg} \mathrm{sec}^{-1}$, or it will cease to radiate X-rays. It should be noted that Johnson (1966) and Burbidge (1967) have reached the same conclusion by similar reasoning. The demonstration has been repeated here to make the comparison with our photometric data more explicit.

One is now immediately struck by the agreement of time scale for energy renewal with the characteristic time of $\sim 300 \mathrm{sec}$ for the optical flickering ( $\$$ II). The similarity seems to us to be so remarkable as to be more than coincidence. If it is not coincidence, one must suppose that the optical fluctuations are directly related to the unknown 
pumping mechanism not only by the time-scale agreement but also by the energetics, following an argument used many years ago by Schwarzschild (1948).

Assume, for the moment, that the optical fluctuations had been strictly phasable and had been due to the pulsation of one of the stellar components. If the velocity amplitude of the pulsation at the photosphere were $\nu$, and if the pulsations were acoustically driving an atmosphere, then the acoustic energy transmitted would be $\sim \rho \nu^{2} V \mathrm{erg} \mathrm{cm}^{-2} \mathrm{sec}^{-1}$, where $\rho$ is the density and $V$ the sound velocity at the interface. If the stellar radius were $r$, then the total energy transmitted would be

$$
P \sim 4 \pi r^{2} \rho \nu^{2} V \text { erg } \sec ^{-1} .
$$

Let us further assume that this acoustic energy can in some unspecified way be used to heat the "corona" to $T \simeq 5 \times 10^{\circ} \mathrm{K}$, perhaps by shocks developing as the waves run down a density gradient.

The input rate must equal the rate of radiation; otherwise, the system would quickly heat or cool, again on the time scale of equation (7). If $\rho \simeq 10^{-65} \mathrm{~g} \mathrm{~cm}^{-3}$ as in the Sun at the base of the photosphere, if $r=10^{11} \mathrm{~cm}$, and if $V=10^{6} \mathrm{~cm} \mathrm{sec}^{-1}$, which is the sound velocity at $T \simeq 5 \times 10^{4^{\circ}} \mathrm{K}$ assumed for the photospheric regions, then $P \simeq 2 \times 10^{37}$ ergs $\mathrm{sec}^{-1}$ if the pulsational velocity amplitude is about $150 \mathrm{~km} \mathrm{sec}-1$. Remarkably enough, this pulsational velocity would produce light variations of about $0.02-\mathrm{mag}$ amplitude in a period of $\sim 300 \mathrm{sec}$ for such a star. It is interesting that this type of calculation does provide the correct order of energy required, using plausible parameters. One is led a second time to suspect the light flickering to be fundamental to the pumping cycle, but the actual case in Sco X-1 is more complicated. The light flickerings are not periodic, but neither are they completely white. Stellar oscillators with such a low $Q$ have not been encountered before except, interestingly enough, in the Sun (Leighton, Noyes, and Simon 1962; Howard 1962, 1967). There is no real evidence from our data that pulsation is, in fact, the cause. Consequently, the mechanism of the flickering is still unknown, and, although the arguments given here may be suggestive, they do not constitute anything like a theory by which the system can be understood. Furthermore, the binary nature of Sco X-1, if substantiated, is likely to be related to the energy pump and to the light flicker in some fundamental way.

These speculations do, however, suggest a crucial experiment. With a time scale as short as equation (7) implies, the response of the $\mathrm{X}$-ray emission to the energy input is fast - so fast, in fact, that changes in the flicker amplitude should change the average $X$ ray emission, and very probably the temperature, in a matter of minutes. An exact correlation between X-ray and optical intensities is, of course, not expected because the mechanical energy input is changed into thermal energy by a noisy process with a finite time constant (e.g., shocks), both of which tend to destroy coherence. ${ }^{1}$

During our five nights of photometric monitoring, the fluctuation amplitudes did change appreciably. The average flicker amplitude was about $0.016 \mathrm{mag}$ on the first four nights, but on June 5 the flicker amplitude dropped a factor of nearly 2, to 0.009 mag. It was small during the entire monitoring period of $2^{\mathrm{h}} 45^{\mathrm{m}}$. It would have been most important to know if the average $X$-ray flux on this night was low by a factor of perhaps 4 (in accordance with eq. [8]) over this $10^{4}$-sec interval. Future extended X-ray observations from balloons or satellites and simultaneous ground-based optical monitoring with adequate photon statistics in short sample times to detect the flicker would seem of prime importance.

${ }^{1}$ This probable lack of coherence in the plasma cloud is, incidentally, one reason why we believe the presence of optical fluctuations in Cyg X-2 and now Sco X-1 demonstrates that the major part of the optical flux in each source may not be optical bremsstrahlung from an extended plasma cloud. A second reason is that optical Sco X-1 changes color as it brightens, which a pure bremsstrahlung source cannot do. 
We are indebted to Edwin Dennison and to the personnel of the Astroelectronics Laboratory for their construction and maintenance of the data readout systems on all telescopes, without which many of the new techniques of data collection would not have been developed. It is a pleasure to thank Guido Münch for discussions on the interstellar Ca II problem. We also had a useful conversation with E. N. Parker on the problem of coronal heating. An early conversation with J. Ostriker and J. E. Hesser at Princeton was useful in suggesting the power of short-sample-time digital recording of stars, and started us thinking about the current problem.

\section{REFERENCES}

Aller, L. H. 1961, The Abundance of Elements (New York: Interscience Publishers), p. 192.

Aller, L. H., Bowen, I. S., and Minkowski, R. 1955, Ap. J., 122, 62.

Aller, L. H., Bowen, I. S., and Wilson, O. C. 1963, Ap. J., 138, 1013.

Babcock, H. W. 1967, Ann. Rept. Dir. Mount Wilson and Palomar Observatories, Carnegie Yrb , No. 66, p. 289.

Beals, C. S., and Oke, J. B. 1953, M.N.R.A.S., 113, 530.

Bertiau, F. C. $1958, A$, .J., 128, 533.

Binnendijk, L. 1952, A p.J., 115, 428.

Braes, L. L. E., and Hovenier, J. W. 1966, Nature, 209, 360

Burbidge, E. M., Lynds, C. R., and Stockton, A. 1967, A p. J. (Letters), 150, L95.

Burbidge, G. R.' 1967, Radio A stronomy and the Galactic System, I.A.U. Symp. 31, ed. H. van Woerden (New York: Academic Press), p. 463.

Elwert, G. 1961, J. Geophys. Res., 66, 391.

Felton, J. E., and Gould, R. J. 1966, Phys. Rev. Letters, 17, 401.

Gorenstein, P., Giacconi, R., and Gursky, H. 1967, Ap.J.' (Letters), 150, L85.

Gorenstein, P., Gursky, H., and Garmire, G. 1968, $A p . J$, 153, 885.

Grader, R. J., Hill, R. W., Seward, F. D., and Toor, A. 1966, Science, 152, 1499.

Grant, G. 1955, Ap.J., 122, 566.

Greene, J. 1959, Ap. J., 130, 693.

Hiltner, W. A., and Mook, D. E. 1967a, Ap.J. (Letters), 150, L23. $1967 b, A p . J ., 150,851$.

Howard, R. 1962, Ap.J., 136, 211. .1967, Solar Phys., 2, 3.

Johnson, H. M. 1966, Ap.J., 146, 960.

Joy, A. H. 1956, Ap. J., 124, 317. . 1961, ibid., 133, 493 .

Joy, A. H., and Swings, P. 1945, A p. J., 102, 353.

Kaler, J. B., Aller, L. H., and Bowen, I S. 1965, A p. J., 141, 912.

Kerr, F. J., and Westerhout, G. 1964, in Galactic Structure, ed. A. Blaauw and M. Schmidt (Chicago: University of Chicago Press), chap. ix, p. 167.

Kraft, R. P. $1962, A p . J ., 135,408$.

.1964, ibid., 139, 457.

Kristian, J., Sandage, A., and Westphal, J. A. 1967, Ap.J. (Letters), 150, L99.

Krzeminski, W. 1962, Pub. A.S.P., 74, 66. . 1965, A p.J., 142, 1051.

Leighton, R. B., Noyes, R. W., and Simon, G. W. 1962, Ap. J., 135, 474.

McGee, R. X., and Murray, J. D. 1961, Australian J. Phys, 14, 260.

McLaughlin, D. B. 1960, in Stellar Atmospheres, ed. J. Greenstein (Chicago: University of Chicago Press), chap. xvii, p. 609.

Mook, D. E. 1967, A p.J. (Letters), 150, L25.

Münch, G 1966, Pub. A.S.P., 78, 305. .1968, in Stars and Stellar Systems, Vol. 7, ed. B. Middlehurst and L. Aller (Chicago: University of Chicago Press), chap. vii.

Mumford, G. S. 1964, Ap.J., 139, 476. . 1966a, ibid., 146, 411

$1966 b$, ibid., p. 962 .

Payne-Gaposchkin, G. 1957, The Galactic Novae (Amsterdam: North-Holland Publishing Co.), pp. 75, 298.

Rossi, B. 1968, Proc. Vetlessen Symp. for J. Oort, ed. L. Woltjer (New York: University of Columbia Press).

Sandage, A., Osmer, P., Giacconi, R., Gorenstein, P., Gursky, H., Waters, J., Bradt, H., Garmire, G., Sreekantan, B. V., Oda, M., Osawa, K., and Jugaku, J. 1966, Ap.J., 146, 316. 


\section{J. A. WESTPHAL, ALLAN SANDAGE, AND JEROME KRISTIAN}

Schmidt, M. 1957, B.A.N., 13, 247.

Schwarzschild, M. 1948, Ap.J., 107, 1.

Spitzer, L. 1949, A p.J., 109, 337.

Stepien, K. 1968, Ap.J. (Letters), 151, L15.

Tucker, W. H., and Gould, R. J. 1966, Ap. J., 144, 244.

Walker, M. F. 1954, Pub. A.S.P., 66, 71.

$$
\text { 1956, A p.J., 123, } 68 .
$$
p. 46.

1957, Non-stable Stars, I.A.U. Symp. 3, ed. G. Herbig (Cambridge: Cambridge University Press),

1958, ibid., 127, 319.

1961, ibid., 134, 171 .

$1963 a$, ibid., $137,485$.

$$
\text { 1963b, ibid., 138, } 313 \text {. }
$$

Wallerstein, G. 1967, Ap. Letters, $1,31$.

Westphal, J. A. 1966, Ap.J. 142, 1661.

Copyright 1968. The University of Chicago. Printed in U.S.A. 